\title{
Knockdown of IncRNA MIAT inhibits proliferation and cisplatin resistance in non-small cell lung cancer cells by increasing miR-184 expression
}

\author{
LONGQIU WU $^{1 *}, \mathrm{CHI} \mathrm{LIU}^{2 *}$ and ZUXIONG ZHANG ${ }^{1}$ \\ ${ }^{1}$ Department of Cardiothoracic Surgery, First Affiliated Hospital of Gannan Medical University, Ganzhou, \\ Jiangxi 341000; ${ }^{2}$ School of Medical and Life Sciences, Chengdu University of \\ Traditional Chinese Medicine, Chengdu, Sichuan 610072, P.R. China
}

Received October 8, 2018; Accepted August 13, 2019

DOI: $10.3892 / \mathrm{ol} .2019 .11084$

\begin{abstract}
Accumulating evidence has demonstrated the important role of long non-coding RNA myocardial infarction-associated transcript (MIAT) in tumorigenesis as a potential oncogene. However, the function of MIAT in non-small cell lung cancer (NSCLC) has yet to be completely elucidated. The present study demonstrated that MIAT expression was significantly upregulated in NSCLC tissues, particularly in aggressive cases, and was highly associated with a poor prognosis. In addition, the upregulated expression of MIAT was observed in cisplatin (CDDP)-resistant H1299 cells. Knockdown of MIAT inhibited the proliferation of NSCLC cells and enhanced the sensitivity of NSCLC cells to CDDP in vitro and in vivo. Further functional analysis demonstrated that MIAT partially exerted its oncogenic effect by upregulating the expression of splicing factor 1 (SF1), by serving as a microRNA (miR)-184 sponge. In conclusion, the present study identified that MIAT functions as a competitive endogenous RNA of miR-184to modulate SF1 expression in NSCLC, which provides a novel insight into the potential therapeutic application of MIAT in NSCLC progression.
\end{abstract}

\section{Introduction}

Lung cancer is the most common cancer worldwide in terms of incidence and mortality rates, and non-small cell lung cancer (NSCLC) is a histological subtype that represents $\sim 85 \%$ of cases (1). Despite advances in diagnosis and treatment,

Correspondence to: Dr Zuxiong Zhang, Department of Cardiothoracic Surgery, First Affiliated Hospital of Gannan Medical University, 23 Youth Road, Ganzhou, Jiangxi 341000, P.R. China E-mail: zuxzhang@163.com

*Contributed equally

Key words: myocardial infarction-associated transcript, miR-184, cisplatin resistance, splicing factor 1 , non-small cell lung cancer at present, patients with NSCLC have a poor prognosis; the overall 5-year survival rate of patients with NSCLC was $<20 \%$ between 2013 and 2018, with high mortality rates due to high rates of invasion, metastasis and postoperative recurrence $(2,3)$. Chemotherapy is an efficient treatment for patients with NSCLC (4). However, frequent multidrug resistance during treatment markedly restricts the curative effect (5). Cisplatin (CDDP) is one of the first-line drugs for NSCLC treatment; however, resistance to CDDP frequently limits its efficacy in a clinical setting (6,7). Therefore, a comprehensive understanding of the etiology and exact molecular mechanism underlying CDDP resistance in NSCLC development and progression is essential to improve the therapeutic efficacy for patients with NSCLC.

Long non-coding RNAs (lncRNAs) are a type of non-encoding RNA transcript of $>200$ nucleotides in length, which do not encode proteins; however, they have the ability to regulate numerous biological processes in tumorigenesis, such as transcriptional and post-trantscriptional regulation, and protein modification $(8,9)$. IncRNAs have attracted large attention due to their critical role in cancer progression (10). In addition, previous studies have hypothesized that lncRNAs may interact with microRNAs (miRNAs) as natural miRNA sponges or competitive endogenous RNAs (ceRNAs) to regulate the expression of target genes, which serves a crucial role in the network of ceRNAs in human diseases $(11,12)$.

Myocardial infarction-associated transcript (MIAT) is a type of lncRNA located on chromosome 22q12.1. It is 30,051 bp in length and was first identified in the year 2000 (13). MIAT was originally reported to be associated with myocardial infarction, and is abundantly expressed in the nervous system and retinal tissue $(14,15)$. Recent studies have suggested that MIAT is upregulated and serves as an oncogene in several types of cancer (16). MIAT is involved in a positive feedback regulatory loop with its transcriptional regulator octamer-binding protein 4 to promote cell proliferation in human malignant mature $B$ cell chronic lymphocytic leukemia and functions as aceRNA of miRNA (miR)-155-5p to regulate dual specificity protein phosphatase 7 in breast cancer $(17,18)$. MIAT was additionally demonstrated to be able to promote NSCLC proliferation and metastasis through the activation of matrix metalloproteinase 
$9(19,20)$. Although a number of previous studies demonstrated a partially oncogenic role of MIAT in NSCLC, the biological mechanisms and miR-sponging role of MIAT in the progression of NSCLC, particularly in CDDP resistance, have yet to be comprehensively elucidated.

The present study hypothesized that MIAT over expression was a characteristic molecular alteration that was highly associated with poor prognosis in patients with NSCLC.

\section{Materials and methods}

Clinical specimens and cell lines. NSCLC tissues and corresponding adjacent normal tissues ( $4 \mathrm{~mm}$ away from the tumor) were collected during therapeutic surgical resection from 60 patients (male, $n=37$; female, $n=23$ ) with NSCLC between May 2017 and February 2019at the First Affiliated Hospital of Gannan Medical University (Ganzhou, China) according to the National Institutes of Health guidelines. The patients underwent surgery without radiotherapy, chemotherapy or any other treatment, and the patient age ranged between 37 and 79 years $(\geq 60$ years, $n=32 ;<60$ years, $n=28$; mean age, $58.88 \pm 12.65$ years). The clinical stage was determined according to the 8th edition of the TNM classification for lung cancer from American Joint Committee on Cancer (21). Tissues were frozen in liquid nitrogen following RNA extraction. The procedures in this study involving animal and human tissue were approved by the Human Ethics Committee of the First Affiliated Hospital of Gannan Medical University. Written informed consent was obtained from each patient prior to the use of their tissues for scientific research. A 2-year follow-up survival survey was conducted; overall survival (OS) was defined as the interval between resection and mortality or the date of last follow-up. The human NSCLC H1299 and CDDP resistant H1299 (H1299-CDDP) cells used in the present study were obtained from the cell bank of Type Culture Collection of the Chinese Academy of Sciences and were cultured in $90 \%$ RPMI-1640 medium (Gibco; Thermo Fisher Scientific, Inc.) supplemented with $10 \%$ fetal bovine serum (Gibco; Thermo Fisher Scientific, Inc.), $1 \%$ penicillin and streptomycin at $37^{\circ} \mathrm{C}$ in a humidified atmosphere containing $5 \% \mathrm{CO}_{2}$.

RNA interference and cell transfection. The small interfering (si)RNA oligonucleotides si-MIAT (5'-GATCCCCGGACA GAGAATGCAAATAATTCAAGAGATTATTTGCATTCT CTGTCCTTTTTA-3') and anti-miR-184 (5'-GCUAGAACU UGUCCUAGCGCUUGGCACAUAUTT-3'), in addition topcDNA3.1-MIAT (MIAT) and miR-184-mimics (miR-184, 5'-TCTACAGTGCCAAGGACTGCACCTCACGTGTCT CCAG-3') were purchased from Shanghai GenePharma Co., Ltd. H1299 and H1299-CDDP cells were transfected with siRNA or negative controls using RNAiMAX Lipofectamine ${ }^{\circledR}$ (Invitrogen; Thermo Fisher Scientific, Inc.) according to the manufacturer's protocol. H1299 Cells were seeded in 6-well plates at a density of $2 \times 10^{5}$ cells/well. In total, $2 \mu \mathrm{g}$ plasmid DNA or 100 pmol siRNA, which was suspended in $50 \mu \mathrm{l}$ Opti-minimum essential medium (MEM) (Invitrogen; Thermo Fisher Scientific, Inc.), was mixed with $10 \mu 1$ Lipofectamine $^{\circledR}$ 2000 (Invitrogen; Thermo Fisher Scientific, Inc.) and left for $20 \mathrm{~min}$ at $25^{\circ} \mathrm{C}$ to form a mixture. Prior to replacing the cell culture medium with $400 \mu \mathrm{l}$ Opti-MEM, H1299 cells were incubated with the mixture at $37^{\circ} \mathrm{C}$ in a humidified atmosphere containing $5 \% \mathrm{CO}_{2}$. After $6 \mathrm{~h}$, the mixture was discarded and the cells were cultured in complete medium at $37^{\circ} \mathrm{C}$ for $24 \mathrm{~h}$. Subsequently, the cells were harvested for reverse transcription-quantitative polymerase chain reaction (RT-qPCR) and western blot analyses.

Dual-luciferase reporter assay. TargetScan (http://www. targetscan.org/) and miRcode (http://www.mircode.org/) were used with bioinformatics databases Starbase v2.0 (http://starbase.sysu.edu.cn/starbase2/), miRcode (http://www.mircode. org/) and TargetScanHuman Release 7.2 (http://www.targetscan. org/vert_72/) to identify the potential target sequences of miR-184. MIAT sequences or splicing factor 1 (SF1) 3'UTR fragments containing corresponding wild-type or mutant-type miR-184 binding site were amplified by PCR and subcloned into pcDNA3.1 luciferase reporter vectors. The pcDNA3.1-MIAT wild-type (WT) or mutant (MUT) and pcDNA3.1-SF1 WT or mutant vectors, with and without designed miR-184 binding sites, were obtained from Shanghai GenePharma Co., Ltd., and co-transfected with miR-184-mimics or negative controls in H1299-CDDP cells using Lipofectamine ${ }^{\circledR} 2000$ (Invitrogen; Thermo Fisher Scientific, Inc.). At 24-48 h following transfection, firefly and Renilla luciferase activities were measured using a Dual-Luciferase Reporter Assay System (Promega Corporation). All values were obtained from at least three independent experiments.

Cell proliferation assay. The cell viability of H1299 and H1299-CDDP cells was assessed by MTT assay. Following incubation of the cells in 96-well plates for $0,12,24,48,72 \mathrm{~h}$, $20 \mu 15 \mathrm{mg} / \mathrm{ml}$ thiazolyl blue in PBS was added to each well, and the plates were incubated at $37^{\circ} \mathrm{C}$ for an additional $2 \mathrm{~h}$. The medium was subsequently removed and the purple colored formazan precipitates were dissolved in $150 \mu \mathrm{l}$ dimethyl sulfoxide. The absorbance was recorded at $490 \mathrm{~nm}$ using a Thermo microplate reader (Rayto Life and Analytical Sciences Co., Ltd.). A colony formation assay was performed, according to a standard protocol (22). H1299 and H1299-CDDP cells were seeded in 6-well plates (2,000 cells/well) and incubated under the aforementioned culture conditions for $\sim 10$ days. Subsequently, cells were fixed with methanol for $15 \mathrm{~min}$ and stained with $0.5 \%$ crystal violet solution for $25 \mathrm{~min}$ at room temperature. Colonies (>50 cells) were counted under a light microscope ( $\mathrm{x} 4$ magnification). The data are presented as the mean of three independent experiments.

Cell apoptosis assay. A cell apoptosis assay was performed with an Annexin V-fluorescein isothiocyanate (FITC) apoptosis detection kit (BD Biosciences) according to the manufacturer's protocol. H1299 and H1299-CDDP cells (1-2×105 cells/well) were seeded in a 6 -well plate for $48 \mathrm{~h}$. Subsequently, cells were harvested and washed twice with ice-cold PBS, and incubated with $5 \mu \mathrm{l}$ Annexin V-FITC and $2 \mu \mathrm{l}$ propidium iodide (BD Biosciences) in $100 \mu \mathrm{l}$ binding buffer for $15 \mathrm{~min}$ at room temperature. The levels of apoptosis were examined using the aforementioned apoptosis detection kit, according to the manufacturer's protocol, by flow cytometry (FACScan; BD Biosciences). The data were analyzed using Cell Quest software (version 5.1; BD Biosciences). 
$R T-q P C R$. Total RNA from transfected H1299-CDDP cells, tumor and adjacent normal tissues was isolated with TRIzol ${ }^{\circledR}$ reagent (Invitrogen; Thermo Fisher Scientific, Inc.) according to the manufacturer's protocol. RT was conducted with random primers using an RT-PCR kit (cat. no. DWA005; Takara Biotechnology Co., Ltd.) at $37^{\circ} \mathrm{C}$ for $15 \mathrm{~min}$ and $85^{\circ} \mathrm{C}$ 15 sec. qPCR was performed with iQ SYBR Green Supermix (Bio-Rad Laboratories, Inc.) according to the manufacturer's protocol. The thermocycling conditions were as follows: $95^{\circ} \mathrm{C}$ for $30 \mathrm{sec}$, followed by 40 cycles of $95^{\circ} \mathrm{C}$ for $5 \mathrm{sec}$ and $60^{\circ} \mathrm{C}$ for $34 \mathrm{sec}$. The primer sequences used were as follows: MIAT, forward: 5'-TCTTCATGTCAGAACACGCTTTA-3' and reverse: 3'-AAGGTCACCCGAGGTCCAA-5'; miR-184 stem loop, 5'-GTCGTATCCAGTGCAGGGTCCGAGGTATTCGC ACTGGATACGACTTCCCA-3', forward, 5'-TGGACGGAG AACTGATAAGGGT-3' and reverse, 3'-CCTTATCAGTTC TCCGTCCATT-5'; and GAPDH forward, 5'-TCTCTGCTC CTCCTGTTC-3' and reverse, 3'-GGTTGAGCACAGGGT ACTTTATTGA-5'. GAPDH mRNA was used as an endogenous control for mRNA. The relative expression level was calculated using the $2^{-\Delta \Delta \mathrm{Cq}}$ method (23) and each experiment was repeated at least three times.

Western blot analysis. Total protein was extracted with Radio-Immunoprecipitation Assay Buffer, quantified by BCA protein detection method (Beyotime Institute of Biotechnology) and separated by $10 \%$ SDS-PAGE using $20 \mu \mathrm{g}$ of protein per lane. The proteins were subsequently transferred to polyvinylidene difluoride (PVDF) membranes (EMD Millipore). The PVDF membranes were blocked with $5 \%$ skim milk at room temperature for 2-4 h. Subsequently, the PVDF membranes were incubated with primary antibodies (all 1:1,000; Abcam) against $\beta$-actin (ab179467), B-cell lymphoma-2 (Bcl-2)-associated X protein (Bax) (ab232479), Bcl-2(ab32124), cleaved caspase-3 (ab2302) and SF1 (ab65815) at $4^{\circ} \mathrm{C}$ overnight. Following incubation with the relevant secondary antibodies (horseradish peroxidase-conjugated goat anti-mouse IgG; 1:5,000; abs20001ss; ABSIN) for $2 \mathrm{~h}$ at $37^{\circ} \mathrm{C}$, the proteins were visualized by autoradiography using enhanced chemiluminescence (Bio-Rad Laboratories, Inc.). The relative expression of the protein of interest was presented as a grayscale ratio of the protein to $\beta$-actin and the results were analyzed using Graph Pad Prism software (GraphPad Software 6.0, Inc.).

In vivo tumor formation assay. A total of 20 male BALB/c nude mice ( 4 weeks old) were obtained from Beijing Huafukang Bioscience Co. Ltd. The in vivo xenograft assay was performed according to guidelines of the Institutional Animal Care and Use Committee (IACUC) and approved by the institutional guidelines of Gannan Medical University. H1299-CDDP cells $\left(1 \times 10^{7}\right.$ cells/mouse $)$ transfected with si-MIAT or si-MIAT negative controls were injected into the right flanks of 30 mice. After 7 days, mice bearing tumors $\sim 100 \mathrm{~mm}^{3}$ were divided into four groups ( $\mathrm{n}=5 \mathrm{mice} / \mathrm{group}$ ): si-NC + PBS, si-NC + CDDP, si-MIAT + PBS and si-MIAT + CDDP. Mice were treated with $\mathrm{CDDP}(4 \mathrm{mg} / \mathrm{kg}$ ) or an equal volume of PBS once every 3 days through tail vein injections, and the tumor size was measured simultaneously using a simplified equation (length $\mathrm{x}$ width ${ }^{2}$ $\mathrm{x} 0.5)$. The weights of the mice were measured every three days following treatment. The mice were sacrificed by cervical dislocation at the humane endpoint (excessive weight loss) and tumors were excised at the end of the treatment period.

Statistical analysis. All experiments were performed at least three times and the data are presented as the mean \pm standard deviation. Significant differences between two groups were analyzed using a Student's t-test (parametric) or Mann-Whitney $\mathrm{U}$ test (non-parametric). Tukey's post-hoc test was performed following one-way ANOVA; least significant difference test was performed following repeated measures ANOVA to analyze tumor size over time. The Kaplan-Meier test was used to estimate OS. The log-rank test was used to analyze the effect of clinical variables and MIAT expression on patients' OS. $\mathrm{P}<0.05$ was considered to indicate a statistically significant difference. The statistical analysis was conducted using Graph Pad software (GraphPad Software 6.0, Inc.).

\section{Results}

MIAT is overexpressed in NSCLC tissues and associated with a poor prognosis. The MIAT expression profile in NSCLC and adjacent normal lung tissues was assessed. In total, 60 pairs of specimens were analyzed by RT-qPCR, and the results demonstrated that the expression of MIAT was significantly $(\mathrm{P}<0.05)$ upregulated in NSCLC tissue compared with that in adjacent normal tissue (Fig. 1A). In addition, the expression of MIAT was revealed to be associated with the metastasis of NSCLC (Fig. 1B). Association analysis additionally demonstrated that the overexpression of MIAT was highly associated with clinical stage (Fig. 1C). Further Kaplan-Meier survival analysis demonstrated that upregulated expression of MIAT was positively associated with a poor 2-year survival of NSCLC (Fig. 1D). Taken together, these results demonstrated that the overexpression of MIAT may be associated with metastasis and a poor prognosis in NSCLC.

Knockdown of MIAT inhibits the proliferation of NSCLC H1299 and H1299-CDDP cells. To examine the role of MIAT in the resistance of NSCLC to CDDP, the expression profile of MIAT in NSCLC H1299 cells and CDDP-resistantH1299 cells was determined. H1299 is a common NSCLC cell line, and the first-line drug for NSCLC in a clinical setting is a platinum compound, which is prone to drug resistance (24). Therefore, the present study investigated and compared H1299 vs. H1299-CDDP cells. Notably, the expression of MIAT in H1299-CDDP was markedly elevated when compared with that in H1299, which suggested that MIAT may be associated with CDDP resistance (Fig. 2A). To investigate the biological role of MIAT in NSCLC, an overexpression plasmid of MIAT (plasmid-MIAT) was transfected into H1299 cells, and siRNA targeting the coding region of MIAT (si-MIAT) was transfected into H1299-CDDP cells. The transfection efficiencies of plasmid-MIAT and si-MIAT were determined by RT-qPCR; the results demonstrated that the expression level of MIAT was decreased in MIAT-siRNA-transfected H1299-CDDP cells and was significantly $(\mathrm{P}<0.001)$ increased in plasmid-MIAT-transfected H1299 cells (Fig. 2B). MTT and colony formation assays demonstrated that transfection with MIAT significantly $(\mathrm{P}<0.001)$ attenuated the proliferation 

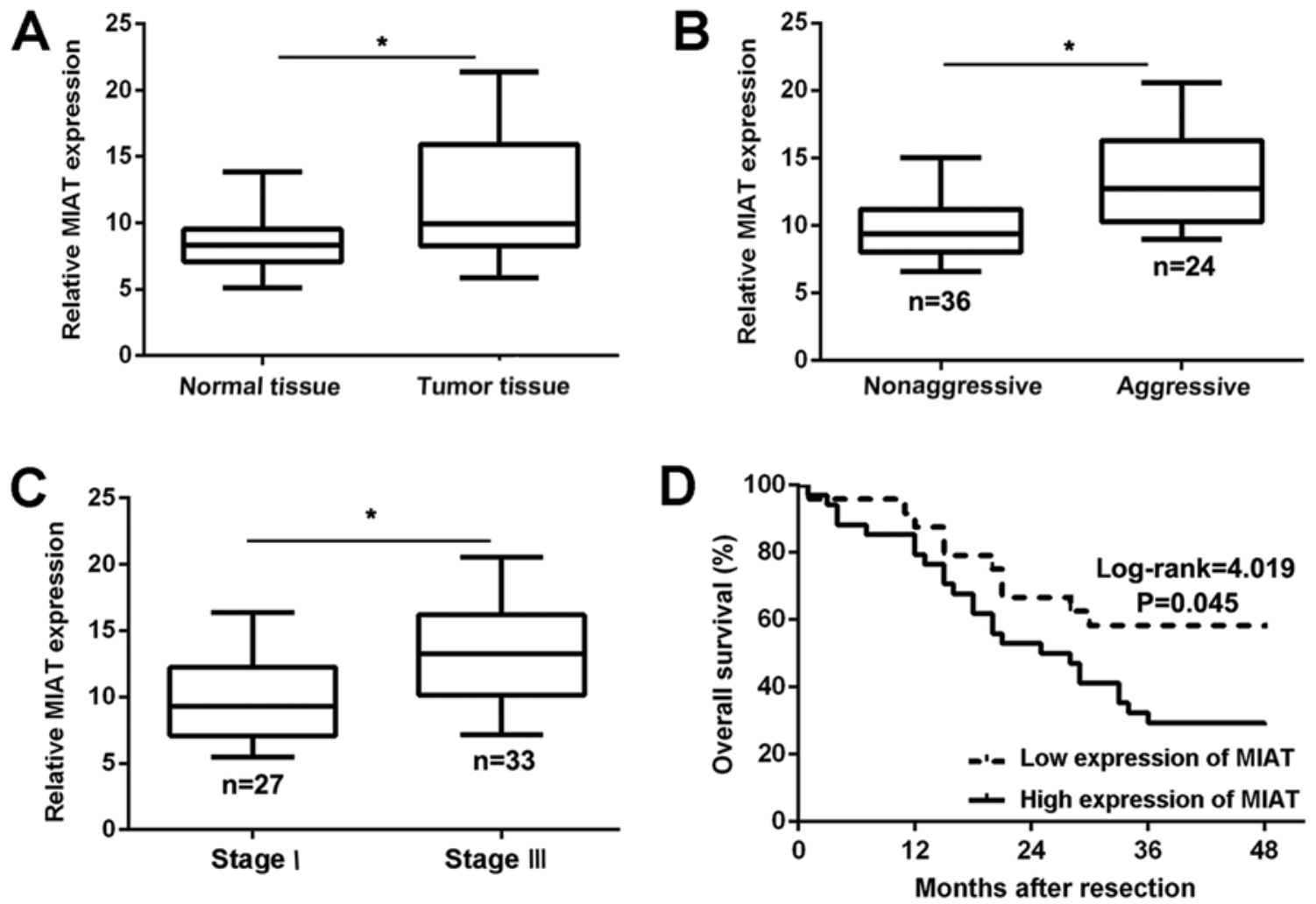

Figure 1. Expression of MIAT in NSCLC tissues. (A) Difference in MIAT expression profile in NSCLC samples (n=60) vs. adjacent normal lung tissues $(\mathrm{n}=60)$. (B) Differential expression of MIAT in patients with NSCLC with metastasis $(\mathrm{n}=24)$ and without metastasis $(\mathrm{n}=36)$. (C) MIAT expression levels in NSCLC were closely associated with TNM stage. (D) Kaplan-Meier analysis of the association between MIAT and overall survival of 60 patients with NSCLC over 48 months. The median level of MIAT was used as the cut-off point between 'High expression of MIAT' and 'Low expression of MIAT'. "P<0.05. MIAT, myocardial infarction-associated transcript; NSCLC, non-small cell lung cancer.

of H1299 and H1299-CDDP cells (Fig. 2C-E). In addition, a flow cytometry was performed to further determine the effect of MIAT on the apoptosis of H1299-CDDP cells, and a significantly $(\mathrm{P}<0.001)$ increased percentage of apoptotic cells in si-MIAT-transfected H1299-CDDP cells was observed (Fig. 2F). Furthermore, apoptotic-associated proteins were analyzed by western blot analysis, which revealed that the silencing of MIAT led to a decrease in the expression level of apoptosis by inhibiting protein $\mathrm{Bcl}-2$ expression, whereas an increased expression level of the pro-apoptotic protein cleaved caspase-3 was observed (Fig. 2G). These data demonstrated that MIAT was able to promote NSCLC cell proliferation.

MIAT sponges miR-184 as a ceRNA. To determine whether MIAT was able to interact with miRs as previously demonstrated, TargetScan (http://www.targetscan.org/) and miRcode (http://www.mircode.org/) were used with bioinformatics databases (Starbase v2.0, miRcode and TargetScan Human Release 7.2). Putative binding sites between MIAT and miR-184 were identified (Fig. 3A). In order to verify the interaction between miR-184 and MIAT at the endogenous level, a dual-luciferase reporter assay was performed in H1299-CDDP cells. The data demonstrated that the luciferase activity was notably decreased when the WT-MIAT-3'untranslated region (3'UTR) and miR-184 were co-transfected into H1299-CDDP cells compared with the activity in H1299-CDDP cells co-transfected with MUT-MIAT-3'UTR and the miR-184 negative control. By contrast, the luciferase activity of
MUT-MIAT-3'UTR exhibited no statistically significant differences (Fig. 3B). In addition, the miR-184 expression level was increased when MIAT was silenced in H1299-CDDP cells (Fig. 3C). Colony formation and MTT assays demonstrated knocking down MIAT expression significantly $(\mathrm{P}<0.001)$ attenuated the proliferation of H1299-CDDP cells. However, co-transfection of si-MIAT and anti-miR-184 into H1299-CDDP cells led to increased proliferation and colony formation compared with that of cells co-transfected with si-MIAT and anti-miR-negative control (Fig. 3D-F). The results suggested that MIAT functions as a ceRNA by sponging miR-184.

MIAT promotes the expression of SF1 by sponging miR-184 in H1299-CDDP cells. Accumulating evidence has suggested that lncRNAs may serve as ceRNAs to regulate the expression of miRs and mRNAs $(25,26)$. Therefore, the present study aimed to elucidate the mechanism underlying the MIAT/miR-184 axis in NSCLC cells. The bioinformatics tool TargetScan revealed that SF1 is a candidate target of miR-184 (Fig. 4A). To validate the interaction between miR-184 and SF1, a dual-luciferase reporter assay was performed in H1299-CDDP cells. The results demonstrated that the luciferase activity was markedly decreased whenWT-SF1-3'UTR and miR-184 were co-transfected into H1299-CDDP cells compared with the activity in H1299-CDDP cells co-transfected with MUT-SF1-3'UTR and miR-184negative control. By contrast, the luciferase activity of MUT-SF1-3'UTR 

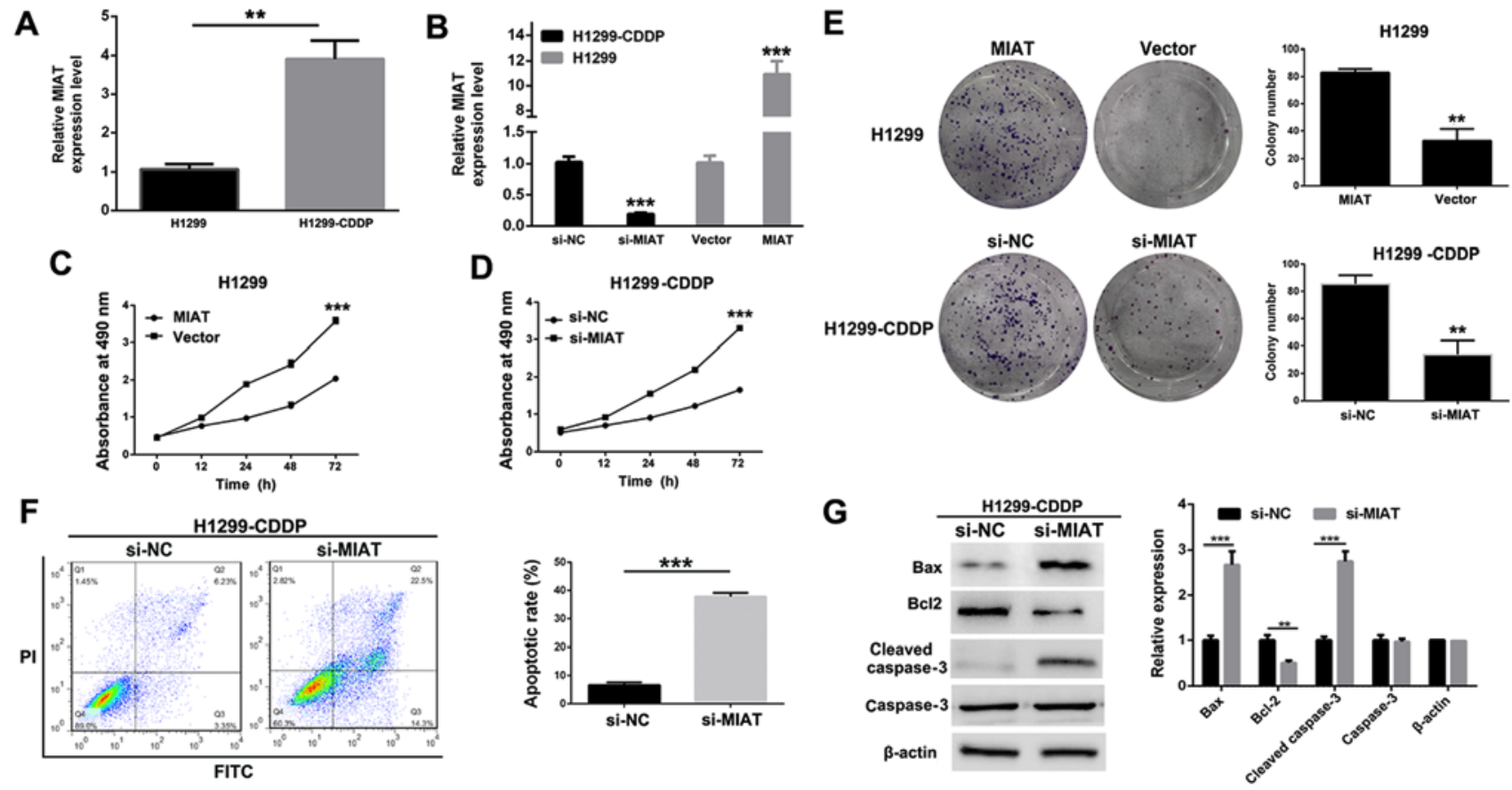

Figure 2. Functional analysis of MIAT in NSCLC cells. (A) The MIAT expression level in H1299 and H1299-CDDP cells was determined by RT-qPCR. (B) The MIAT expression level in H1299-CDDP cells transfected with si-MIAT or NC-siRNA, as well as in H1299 cells transfected with pcDNA3.1-MIAT plasmid (MIAT) or empty vector was determined by RT-qPCR. (C and D) Growth curves of (C) H1299 cells transfected with MIAT or vector and (D) H1299-CDDP cells transfected with si-MIAT or si-NC were determined via MTT assays. (E) Colony formation assays of H1299 cells transfected with MIAT or vector and H1299-CDDP cells transfected with si-MIAT or si-NC were performed. (F) Flow cytometry assays were performed to analyze the apoptosis of H1299-CDDP cells following treatment with si-MIAT or si-NC. (G) The levels of Bax, Bcl-2 and cleaved caspase-3 following MIAT silencing in H1299-CDDP cells were determined via western blotting. ${ }^{*} \mathrm{P}<0.05,{ }^{* *} \mathrm{P}<0.01$ and ${ }^{* * *} \mathrm{P}<0.001$. MIAT, myocardial infarction-associated transcript; NSCLC, non-small cell lung cancer; NC, negative control; si, small interfering; CDDP, cisplatin; RT-qPCR, reverse transcription-quantitative polymerase chain reaction; Bcl-2, B-cell lymphoma-2; Bax, Bcl-2-associated X protein.

exhibited no statistically significant differences (Fig. 4B). Furthermore, western blot analysis demonstrated that the overexpression of miR-184led to a markedly decreased expression level of SF1 compared with that of miR-184 negative control; by contrast, SF1expression was markedly increased when miR-184 was silenced by anti-miR-184 compared with that caused byanti-miR-184 negative control in H1299-CDDP cells (Fig. 4C and D). Overexpression of MIAT additionally induced a notably increased expression level of SF1 (Fig. 4E and F). Although MIAT + miR-NC upregulated the expression of SF1, the expression level of SF1 in cells treated with MIAT + miR-184 was significantly downregulated compared with that in cells treated with MIAT. These results suggested that upregulated MIAT acts as a ceRNA of miR-184 to regulate SF1 expression.

Knockdown of MIAT suppresses tumor growth and enhances the sensitivity of NSCLC to CDDP in nude mice. In order to examine the association between MIAT and CDDP resistance in vivo, a subcutaneous xenograft tumor model in nude mice was established. H1299-CDDP cells transfected with si-MIAT or a si-MIAT negative control were subcutaneously injected into the right flanks of the nude mice. The results demonstrated that treatment with CDDP or MIAT knockdown markedly attenuated the tumor growth and tumor volume (Fig. 5A and B), and the tumor weight was also decreased (Fig. 5C). In addition, silencing MIAT further intensified the repression efficacy of CDDP on tumor growth. Taken together, these data suggested that knockdown of MIAT arrested tumor growth and increased CDDP sensitivity in NSCLC.

\section{Discussion}

Accumulating evidence has demonstrated that lncRNAs exert complex effects in cancer progression $(27,28)$. Abnormally expressed lncRNAs have been recognized to exert oncogenic properties in NSCLC, for instance, upregulated lncRNA maternally expressed 3 was observed to inhibit NSCLC cell proliferation and induce apoptosis by affecting p53 expression (29). In addition, a previous study demonstrated that overexpressed lncRNA sprout RTK signaling antagonist 4-intronic transcript 1 promoted NSCLC cell proliferation and metastasis by affecting the epithelial-mesenchymal transition via histone-lysine $\mathrm{N}$-methyltransferase enhancer of zeste 2 (30). Aberrant expression of microRNAs is associated with tumor progression and metastasis $(31,32)$. Drug resistance remains a principal challenge in chemotherapy, since it impedes the curative effect when administered to patients (7). MIAT has been recognized as a potent oncogene in breast cancer (17), chronic lymphocytic leukemia (18) and colorectal cancer (33). In the present study, a significant overexpression of MIAT was observed in NSCLC tissues compared with that in adjacent normal lung tissues. In addition, the expression level of MIAT in patients with NSCLC was significantly associated with increased aggressiveness, a lower OS rate and advanced clinical stage. Furthermore, the expression of 

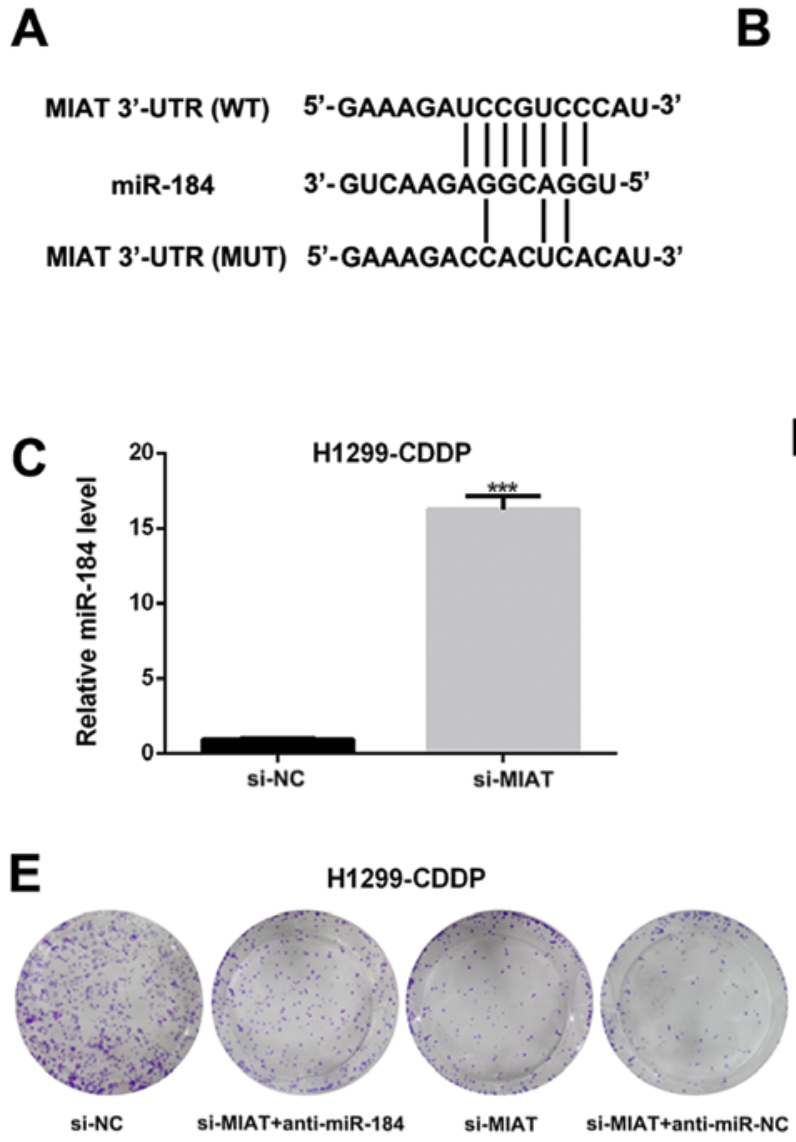

B
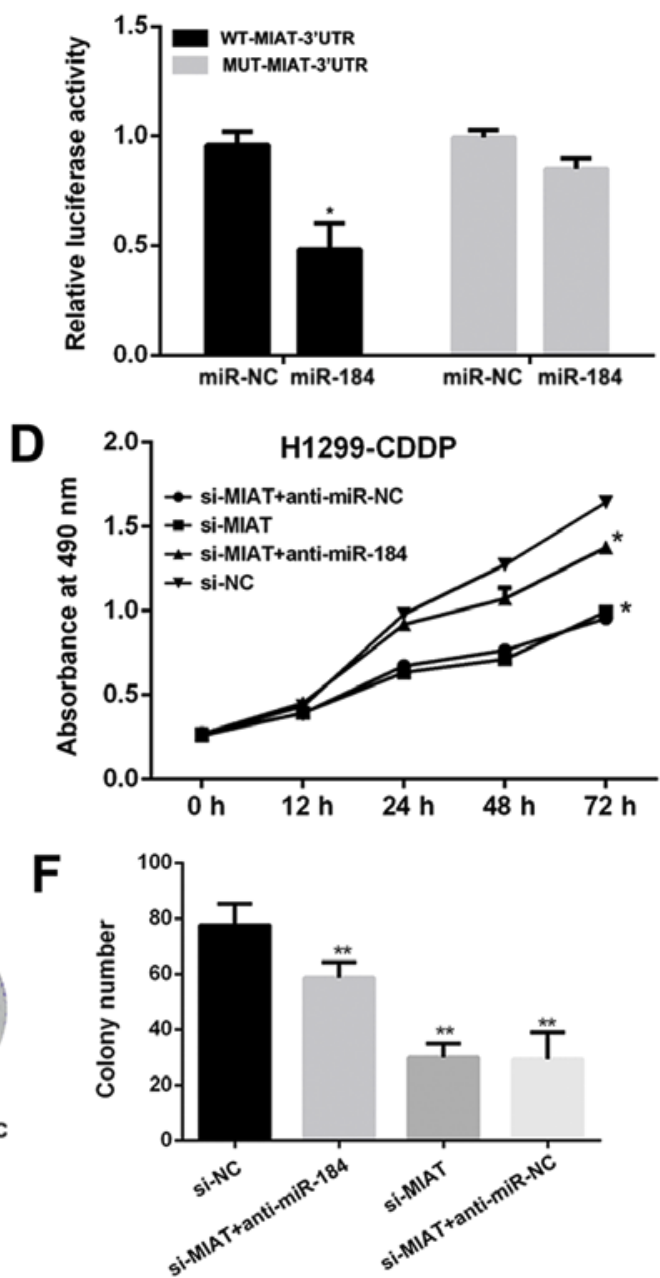

Figure 3. MIAT acts as a competitive endogenous RNA by sponging miR-184. (A) The predicted binding sites of MIAT to miR-184 sequence were illustrated. (B) The luciferase activity of H1299-CDDP cells co-transfected with miR-184 mimics and luciferase reporters containing WT-MIAT-3'-UTR or MUT-MIAT-3'-UTR transcripts were analyzed. (C) The relative miR-184 level in H1299-CDDP cells transfected with si-MIAT or si-NC was determined by reverse transcription-quantitative polymerase chain reaction. (D) Growth curves of H1299-CDDP cells transfected with si-MIAT + anti-miR-NC, si-MIAT, si-MIAT + anti-miR-184 or si-NC were determined via MTT assays. (E) Images of colony formation assays of H1299-CDDP cells transfected with si-MIAT + anti-miR-NC, si-MIAT, si-MIAT + anti-miR-184 or si-NC were performed. (F) The statistical analysis of colony formation assays of H1299-CDDP cells transfected with si-MIAT + anti-miR-NC, si-MIAT, si-MIAT + anti-miR-184 or si-NC. ${ }^{*} \mathrm{P}<0.05,{ }^{* *} \mathrm{P}<0.01$ and ${ }^{* * *} \mathrm{P}<0.001$ vs. negative control. MIAT, myocardial infarction-associated transcript; NSCLC, non-small cell lung cancer; NC, negative control; si, small interfering; WT, wild type; MUT, mutant; URT, untranslated region; CDDP, cisplatin; miR, microRNA.

MIAT in H1299-CDDP cells was increased compared with that in H1299 cells. Knockdown experiments identified the tumorigenic role of MIAT in promoting cellular proliferation in NSCLC cells. Furthermore, the in vivo assay demonstrated that the silencing of MIAT was able to inhibit tumor growth and enhance the sensitivity of NSCLC to CDDP.

A ceRNA hypothesis suggested that lncRNAs may interact with miRs directly in order to regulate the expression of target genes, and a novel regulatory network has been identified in the crosstalk between IncRNAs and miRs (11). IncRNA MIAT has been recognized as a sponge of miR-155-5p to promote breast cancer progression by regulating dual specificity protein phosphatase expression (17). Knockdown of MIAT was able to inhibit breast cancer cell proliferation, migration and invasion by sponging miR-155-5p (17). Therefore, the present study used TargetScan to identify the putative target miR of MIAT. Putative binding sites were identified between MIAT and miR-184. The endogenous interaction between MIAT and miR-184 was revealed by a dual-luciferase assay, and the miR-184 expression levels were restored as MIAT was silenced. This further verified that MIAT served as a ceRNA by sponging miR-184. In addition, it was identified that SF1 is a target gene of the MIAT/miR-184 axis in NSCLC cells using bioinformatics analysis, which was subsequently demonstrated by dual-luciferase reporter assays. SF1 is an RNA-binding protein, which is involved in the spliceosome-mediated assembly of specific pre-mRNAs (13). Previous studies identified a correlation between SF1 and cancer. For example, SF1 was able to promote the tumorigenesis of testicular germ cell tumors $(34,35)$. In the present study, it was observed that the expression levels of SF1 were regulated by MIAT by sponging miR-184 in NSCLC cells. However, additional studies are required to examine the function and molecular mechanism of SF1 in various types of cancer. The in vivo analysis of the present study further validated that depletion of MIAT was able to suppress tumor growth and enhance the sensitivity of NSCLC to CDDP. These results suggested that inhibiting the expression of MIAT may be a potential therapeutic strategy 
A

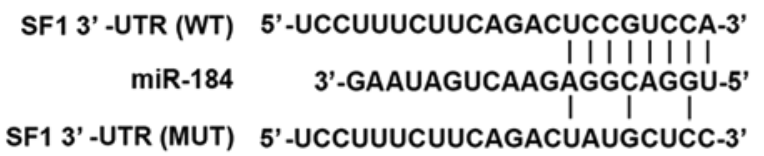

C

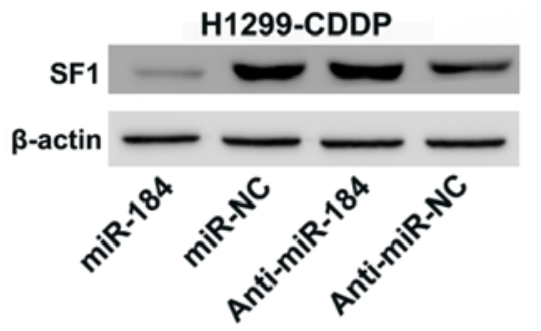

$\mathbf{E}$

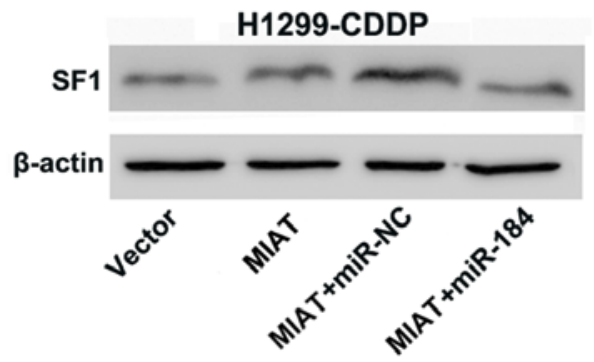

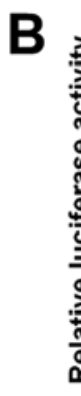
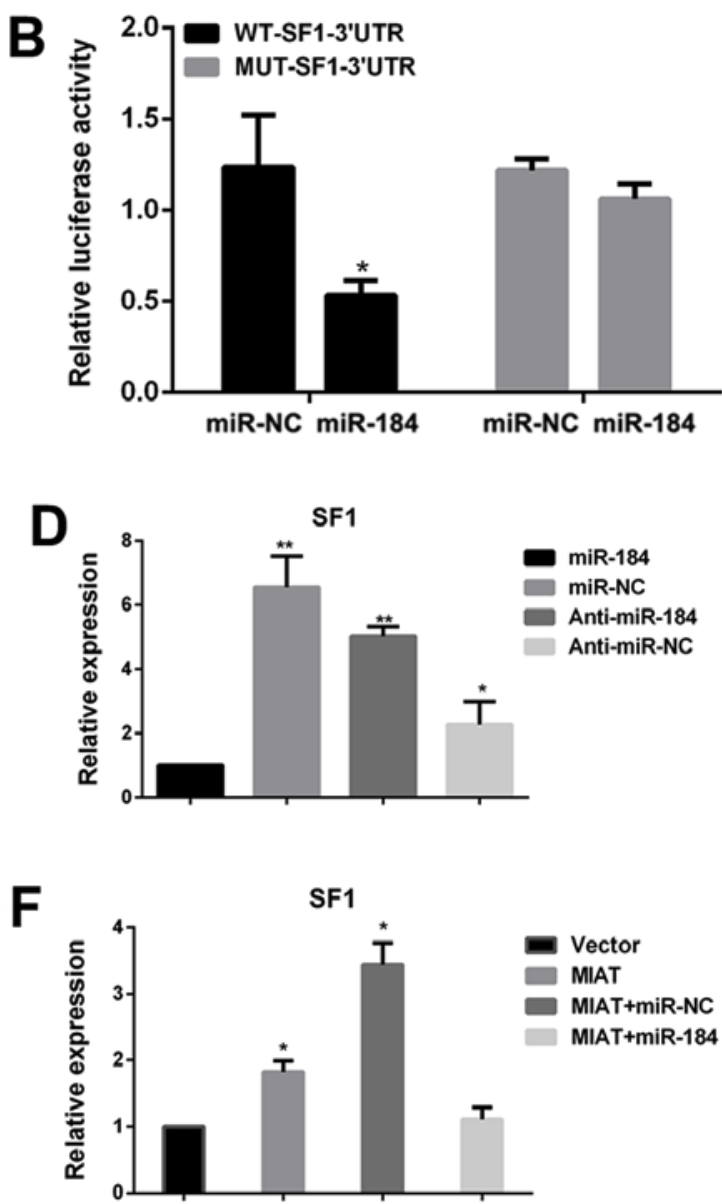

Figure 4. MIAT-mediated sponging of miR-184 elevates the expression of SF1. (A) Putative binding sites and corresponding mutant region for SF1 within miR-184. (B) The effect of miR-184 on the luciferase activity of WT-SF1-3'UTR and MUT-SF1-3'UTR reporter systems was measured by a luciferase reporter assay in H1299-CDDP cells. (C-F) The effects of overexpression or knockdown of miR-184 or MIAT on the expression of SF1 were detected in H1299-CDDP cells by western blotting. ${ }^{*} \mathrm{P}<0.05$ and ${ }^{* *} \mathrm{P}<0.01$ vs. the corresponding negative control. MIAT, myocardial infarction-associated transcript; WT, wild type; MUT, mutant; URT, untranslated region; SF1, splicing factor 1; CDDP, cisplatin; miR, microRNA.

A

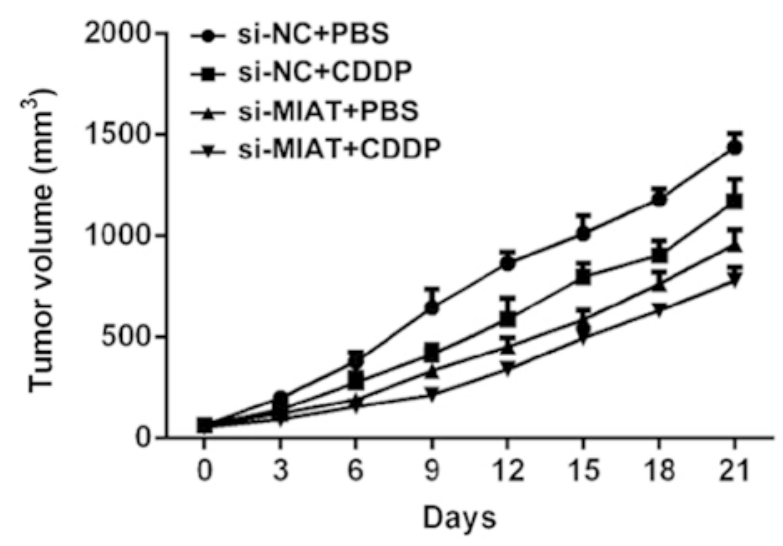

B

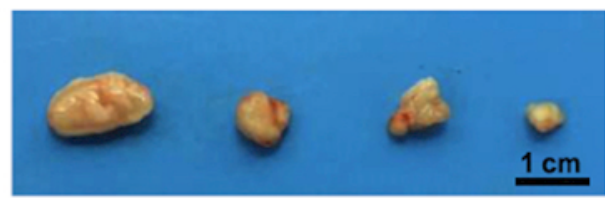

C

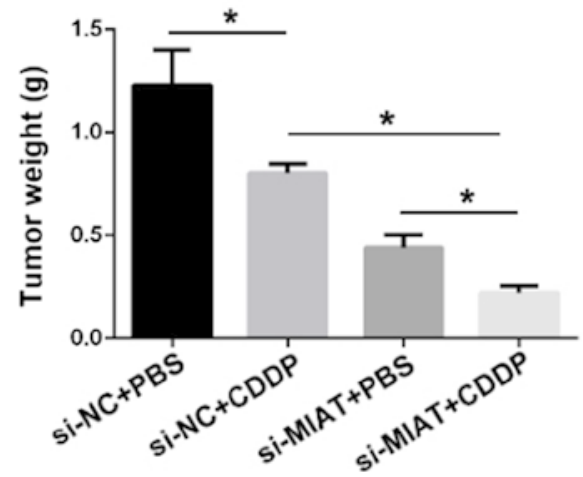

Figure 5. MIAT knockdown suppresses tumor growth and enhances the sensitivity of non-small cell lung cancer to CDDP in vivo. (A) Tumor volume curve of mice following CDDP treatment. (B) Tumors collected from mice. (C) Tumor weight was measured. * $\mathrm{P}<0.05$. MIAT, myocardial infarction-associated transcript; NC, negative control; si, small interfering; CDDP, cisplatin.

to promote therapeutic activity and enhance CDDP susceptibility in NSCLC.
In summary, overexpressed MIAT in CDDP-resistant H1299 cells and NSCLC tissue specimens promoted 
proliferation and enhanced resistance to CDDP in NSCLC cells in vitro and in vivo, which suggests that MIAT exerts oncogenic properties in NSCLC. The biological functional analysis demonstrated that MIAT exerted its oncogenic effect partially by upregulating the expression of SF1 as a miR-184 sponge. The results of the present study provided novel insights into the molecular signaling network involved in NSCLC carcinogenesis and CDDP resistance, and demonstrated the potential therapeutic application of MIAT in NSCLC progression.

\section{Acknowledgements}

Not applicable.

\section{Funding}

The present study was supported by the Science and Technology research project of the Department of Education of Jiangxi Province (grant no. GJJ170099).

\section{Availability of data and materials}

The datasets used and/or analyzed during the present study are available from the corresponding authors on reasonable request.

\section{Authors' contributions}

LW conceived and designed the study. CL acquired and analyzed the data. LW and ZZ interpreted the data and wrote the manuscript. ZZ critically revised the manuscript. All authors read and approved the final version of the manuscript.

\section{Ethics approval and consent to participate}

The present study was approved by the Human Ethics Committee of the First Affiliated Hospital of Gannan Medical University. Written informed consent was obtained from all patients prior to using their tissues for scientific research.

\section{Patient consent for publication}

Not applicable.

\section{Competing interests}

The authors declare that they have no competing interests.

\section{References}

1. Youlden DR, Cramb SM and Baade PD: The international epidemiology of lung cancer-Geographical distribution and secular trends. J Thorac Oncol 3: 819-831, 2008.

2. Reck M,Heigener DF, Mok T, Soria JC and Rabe KF: Management of non-small-cell lung cancer: Recent developments. Lancet 382: 709-719, 2013.

3. Molina JR, Yang P, Cassivi SD, Schild SE and Adjei AA: Non-small cell lung cancer: Epidemiology, risk factors, treatment, and survivorship. Mayo Clin Proc 83: 584-594, 2008.

4. Duma N, Santana-Davila R and Molina JR: Non-small cell lung cancer: Epidemiology, screening, diagnosis, and treatment. Mayo Clin Proc 94: 1623-1640, 2019
5. Gottesman MM, Lavi O, Hall MD and Gillet JP: Toward a better understanding of the complexity of cancer drug resistance. Annu Rev Pharmacol Toxicol 56: 85-102, 2016.

6. Fennell DA, Summers Y, Cadranel J, Benepal T, Christoph DC, Lal R, Das M, Maxwell F, Visseren-Grul C and Ferry D: Cisplatin in the modern era: The backbone of first-line chemotherapy for non-small cell lung cancer. Cancer Treat Rev 44: 42-50, 2016.

7. Galluzzi L, Vitale I, Michels J, Brenner C, Szabadkai G, Harel-Bellan A, Castedo M and Kroemer G: Systems biology of cisplatin resistance: Past, present and future. Cell Death Dis 5: e1257, 2014.

8. Esteller M: Non-coding RNAs in human disease. Nat Rev Genet 12: 861-874, 2011

9. Shi X, Sun M, Liu H, Yao Y and Song Y: Long non-coding RNAs: A new frontier in the study of human diseases. Cancer Lett 339: 159-166, 2013.

10. Prensner JR and Chinnaiyan AM: The Emergence of lncRNAs in cancer biology. Cancer Discov 1: 391-407, 2011.

11. Anastasiadou E, Jacob LS and Slack FJ: Non-coding RNA networks in cancer. Nat Rev Cancer 18: 5-18, 2018.

12. Liz $\mathrm{J}$ and Esteller M: IncRNAs and microRNAs with a role in cancer development. Biochim Biophys Acta 1859: 169-176, 2016.

13. Ishii N, Ozaki K, Sato H, Mizuno H, Saito S, Takahashi A, Miyamoto Y, Ikegawa S, Kamatani N, Hori M, et al: Identification of a novel non-coding RNA, MIAT, that confers risk of myocardial infarction. J Hum Genet 51: 1087-1099, 2006.

14. Liao J, He Q, Li M, Chen Y, Liu Y and Wang J: LncRNA MIAT: Myocardial infarction associated and more. Gene 578: 158-161, 2016.

15. Sun C, Huang L, Li Z, Leng K, Xu Y, Jiang X and Cui Y: Long non-coding RNA MIAT in development and disease: A new player in an old game. J Biomed Sci 25: 23, 2018.

16. Wang R, Zhao L, Ji L, Bai L and Wen Q: Myocardial infarction associated transcript (MIAT) promotes papillary thyroid cancer progression via sponging miR-212. Biomed Pharmacother 118: $109298,2019$.

17. Luan T, Zhang X, Wang S, Song Y, Zhou S, Lin J, An W, Yuan W, Yang Y, Cai H, et al: Long non-coding RNA MIAT promotes breast cancer progression and functions as ceRNA to regulate DUSP7 expression by sponging miR-155-5p. Oncotarget 8 : 76153-76164, 2017.

18. Sattari A, Siddiqui H, Moshiri F, Ngankeu A, Nakamura T, Kipps TJ and Croce CM: Upregulation of long noncoding RNA MIAT in aggressive form of chronic lymphocytic leukemias. Oncotarget 7: 54174-54182, 2016.

19. Lai IL, Yang CA, Lin PC, Chan WL, Lee YT, Yen JC, Chang YS and Chang JG: Long noncoding RNA MIAT promotes non-small cell lung cancer proliferation and metastasis through MMP9 activation. Oncotarget 8: 98148-98162, 2017.

20. Zhang HY, Zheng FS, Yang W and Lu JB: The long non-coding RNA MIAT regulates zinc finger E-box binding homeobox 1 expression by sponging miR-150 and promoteing cell invasion in non-small-cell lung cancer. Gene 633: 61-65, 2017.

21. Shirasawa M, Fukui T, Kusuhara S, Hiyoshi Y, Ishihara M, Kasajima M, Nakahara Y, Otani S, Igawa S, et al: Prognostic significance of the 8th edition of the TNM classification for patients with extensive disease small cell lung cancer. Cancer Manag Res 10: 6039-6047, 2018.

22. Zeng A, Hua H, Liu L and Zhao J: Betulinic acid induces apoptosis and inhibits metastasis of human colorectal cancer cells in vitro and in vivo. Bioorg Med Chem 27: 2546-2552, 2019.

23. Livak KJ and Schmittgen TD: Analysis of relative gene expression data using real-time quantitative PCR and the 2(-Delta Delta C(T)) method. Methods 25: 402-408, 2001.

24. Faehling M, Achenbach J, Staib P, Steffen U, Tessen HW, Gaillard VE and Brugger W: Erlotinib in routine clinical practice for first-line maintenance therapy in patients with advanced non-small cell lung cancer (NSCLC). J Cancer Res Clin Oncol 144: 1375-1383, 2018.

25. Tu L, Huang Q, Fu S and Liu D: Aberrantly expressed long noncoding RNAs in hypertrophic scar fibroblasts in vitro: A microarray study. Int J Mol Med 41: 1917-1930, 2018.

26. Zhou RS, Zhang EX, Sun QF, Ye ZJ, Liu JW, Zhou DH and Tang Y: Integrated analysis of IncRNA-miRNA-mRNA ceRNA network in squamous cell carcinoma of tongue. BMC Cancer 19: $779,2019$.

27. Zhang CG, Yin DD, Sun SY and Han L: The use of lncRNA analysis for stratification management of prognostic risk in patients with NSCLC. Eur Rev Med Pharmacol Sci 21: 115-119, 2017. 
28. Zhu H, Yu J,Zhu H, Guo Y and Feng S: Identification of key lncRNAs in colorectal cancer progression based on associated protein-protein interaction analysis. World J Surg Oncol 15: 153, 2017.

29. Lu KH, Li W, Liu XH, Sun M, Zhang Ml, Wu WQ, Xie WP and Hou YY: Long non-coding RNA MEG3 inhibits NSCLC cells proliferation and induces apoptosis by affecting p53 expression. BMC Cancer 13: 461, 2013.

30. Sun M, Liu XH, Lu KH, Nie FQ, Xia R, Kong R, Yang JS, Xu TP, Liu YW, Zou YF, et al: EZH2-mediated epigenetic suppression of long noncoding RNA SPRY4-IT1 promotes NSCLC cell proliferation and metastasis by affecting the epithelial-mesenchymal transition. Cell Death Dis 5: e1298, 2014.

31. Ma YJ, Ha CF, Bai ZM, Li HN, Xiong Y and Jiang J: Overexpression of microRNA-205 predicts lymph node metastasis and indicates an unfavorable prognosis in endometrial cancer. Oncol Lett 12: 4403-4410, 2016.
32. Pradhan AK, Emdad L, Das SK, Sarkar D and Fisher PB: The enigma of miRNA regulation in cancer. Adv Cancer Res 135: $25-52,2017$.

33. Liu Z, Wang H, Cai H, Hong Y, Li Y, Su D and Fan Z: Long non-coding RNA MIAT promotes growth and metastasis of colorectal cancer cells through regulation of miR-132/Derlin-1 pathway. Cancer Cell Int 18: 59, 2018.

34. Zhu R, Heaney J, Nadeau JH, Ali S and Matin A: Deficiency of splicing Factor 1 suppresses the occurrence of testicular germ cell tumors. Cancer Res 70: 7264-7272, 2010.

35. Shitashige $M$, Naishiro $Y$, Idogawa $M$, Honda $K$, Ono $M$, Hirohashi S and Yamada T: Involvement of Splicing Factor-1 in beta-catenin/T-Cell Factor-4-mediated gene transactivation and Pre-mRNA splicing. Gastroenterology 132: 1039-1054, 2007. 